\title{
THE INTERPRETATION OF ELLIPSOMETRIC MEASUREMENTS OF ION BOMBARDMENT OF NOBLE GASES ON SEMICONDUCTOR SURFACES *
}

\author{
A.H.M. HOLTSLAG, U.C. SLAGER and A. VAN SILFHOUT \\ Department of Applied Physics, Twente University of Technology, P.O. Box 217, 7500 AE Enschede, \\ The Netherlands
}

Received 1 April 1984

Low energy noble gas ion bombardment and thermal desorption studies were carried out on $\mathrm{Si}(111)$ and analysed, in situ, using spectroscopic ellipsometry. The amorphous layer thickness and implanted noble gas fraction were calculated.

\section{Introduction}

The influence of ion bombardment on the structure of the silicon lattice has been the subject of numerous investigations. The various effects induced by low energy ion bombardment in the outer layers of a solid - lattice damage, ion implantation, surface roughness, sputtering and surface state changes - are reflected in changes of the complex dielectric constant $\tilde{\boldsymbol{\epsilon}}$, which can be measured by means of spectroscopic ellipsometry [1-3].

This paper reports noble gas ion bombardment and desorption studies carried out on $\mathrm{Si}(111)$ and analysed using spectroscopic ellipsometry.

\section{Experimental}

The experiments were carried out in an UHV system (base pressure $10^{-8}$ $\mathrm{Pa}$ ), having facilities for sample temperature control, spectroscopic PSRA ellipsometry, AES, mass selective ion bombardment, sputtering and mass

\footnotetext{
* This work is part of the research program of the Stichting voor Fundamenteel Onderzoek der Materie (Foundation for Fundamental Research on Matter) and was made possible by financial support from the Nederlandse Organisatie voor Wetenschappelijk Onderzoek (Netherlands Organisation for the Advancement of Pure Research).
} 
spectrometry. A minicomputer system has been constructed wich automatically controls the ellipsometer and data acquisition [4]. Initially the ellipsometer was calibrated [5] and alligned (angle of incidence $70.25^{\circ}$ ). Every ellipsometric measurement $(\Delta, \psi)$ was preceded by an offset measurement at each analyser setting, since during each desorption the silicon sample is heated up to $800^{\circ} \mathrm{C}$, resulting in the optical measurements heing disturbed by the partially polarised light emitted from the sample.

Before bombardment the silicon sample was cleaned by argon ion sputtering $\left(45^{\circ}, 800 \mathrm{eV}, 2 \times 10^{-6} \mathrm{~A} \mathrm{~cm}^{-2}\right)$ followed by a 30 min anneal at $800^{\circ} \mathrm{C}$ and AES. The ion bombardments were carried out using a $2 \mathrm{keV}$ mass selective ion beam, at normal incidence, for two different fluxes (flux 1: $15 \times 10^{-9} \mathrm{~A} \mathrm{~cm}^{-2}$; flux 2: $115 \times 10^{-9} \mathrm{~A} \mathrm{~cm}^{-2}$ ).

The intensity and profile of the beam was measured using a Faraday cup. After each bombardment a desorption measurement was performed. Using a controllable power supply the sample was directly heated in 50 reproducible increments from $25^{\circ}$ to $800^{\circ} \mathrm{C}$. For $100 \mathrm{~s}$ after each increment the sample temperature and the partial pressure, $p(t)$, of the previously implanted noble gas were measured, followed by determination of $\Delta$ and $\psi$ at four wavelengths (and $p, T$ ) (A, B, C and D: 695, 595, 495 and $395 \mathrm{~nm}$ ), during $200 \mathrm{~s}$.

For each desorption the total amount of desorbed particles per unit surface area, $N_{\mathrm{D}}(t)$, was calculated from:

$\frac{N_{\mathrm{D}}(t)}{N_{\text {ref }}}=\frac{p(t)-p(0)}{P_{\text {ref }}}+\frac{1}{\tau} \int_{0}^{t} \frac{p\left(t^{\prime}\right)-p(0)}{P_{\text {ref }}} \mathrm{d} t^{\prime}, . N_{\text {ref }}=\frac{V P_{\text {ref }}}{k T_{\mathrm{v}} A_{\text {eff }}}$,

where $T_{v}$ is the vessel temperature, $A_{\text {eff }}$ is the effective implanted area and $\tau$ is the quotient of the UHV vessel volume $V$ and the effective pumping speed $S$ $(\tau=62.5 \mathrm{~s}$ for argon).

\section{Results and discussion}

\subsection{Optical effects of noble ion gas bombardments on Si(111)}

The optical parameters $\delta \Delta$ and $\delta \psi$, as shown in the figures, are defined as $\delta \Delta=\Delta(N, T, \lambda)-\bar{\Delta}(\mathrm{T}, \lambda)$ and $\delta \psi=\psi(N, T, \lambda)-\bar{\psi}(T, \lambda)$ where $\bar{\Delta}(T, \lambda)$ and $\bar{\psi}(T, \lambda)$ refer to the clean substrate and from which the dielectric constant $\tilde{\epsilon}_{\mathrm{c}}(T, \lambda)$ of c-silicon can be calculated. $\Delta(N, T, \lambda)$ and $\psi(N, T, \lambda)$ refer to the bombardment substrate containing a dose $N$ at temperature $T$. In figs. $1 \mathrm{~A}$ and $1 \mathrm{~B}$ the measured $\delta \psi$ and $\delta \Delta$ are given. In fig. 1B the effect of the bombardment can be divided into four different characteristic regions having divisions at $\mathrm{Q}, \mathrm{R}, \mathrm{S}$ and $\mathrm{T}\left(1.37 \AA^{-2}\right)$.

From $\mathrm{S}$ to $\mathrm{T}, \delta \Delta$ changes slowly with increasing $N$; after dose $\mathrm{T}, \delta \Delta$ and $\delta \psi$ 


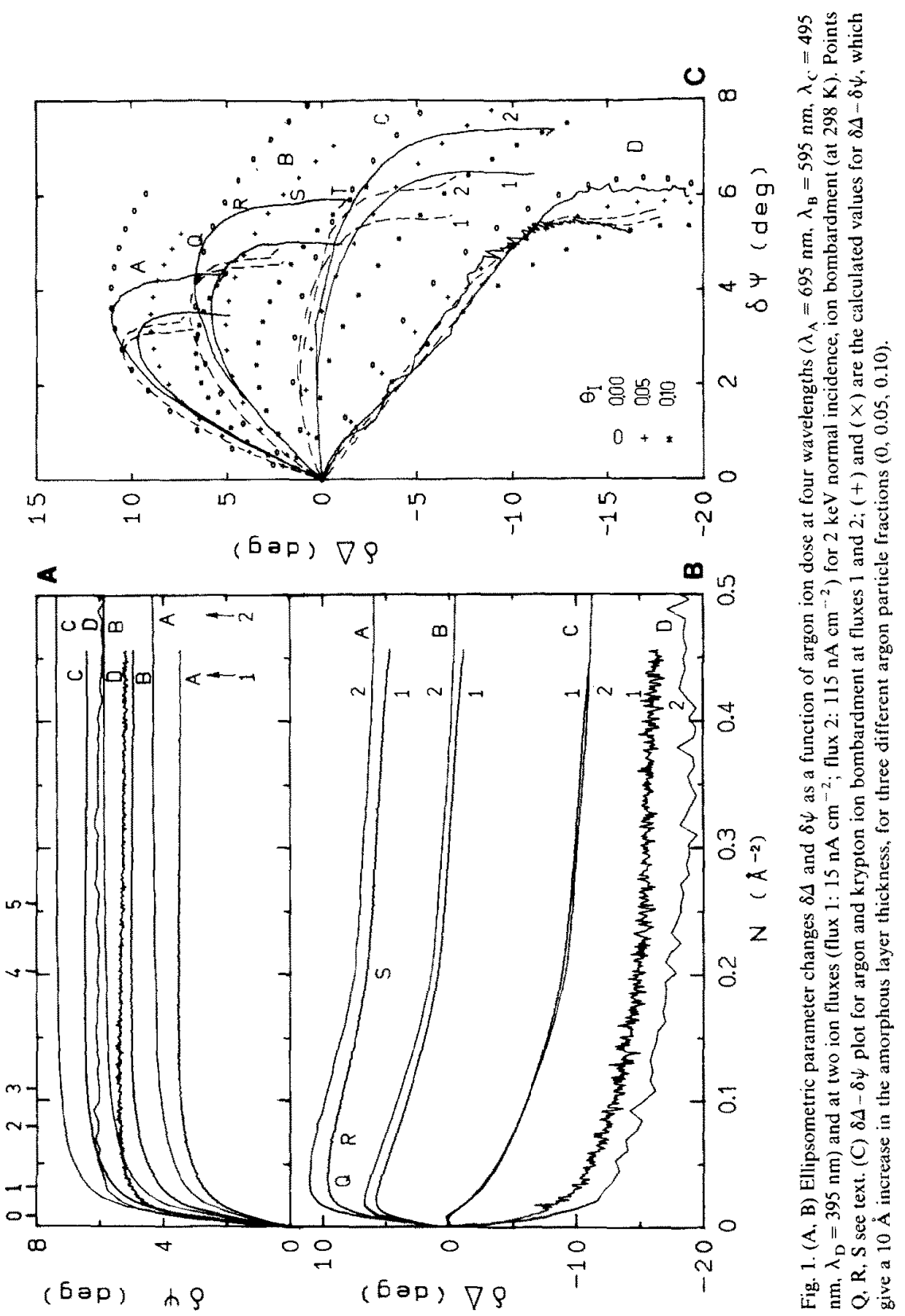


remain constant. Upon termination of every bombardment $\delta \Delta$ increases and $\delta \psi$ decreases (see fig. 3). From point $\mathrm{R}$ the optical change in $\delta \Delta$ is scalable, meaning that $\delta \Delta\left(N, T_{0}, \lambda_{k}\right)=a_{k l}+b_{k l} \delta \Delta\left(N, T_{0}, \lambda_{l}\right)$ where $\lambda_{k}$ and $\lambda_{l}$ stand for two different wavelengths. However, this relationship does not hold for the region $0 \mathrm{QR}$, suggesting that we can separate at least two optical processes caused by bombardment. In fig. $1 \mathrm{C}$ a $\delta \Delta-\delta \psi$ plot is given for the $2 \mathrm{keV}$ argon and krypton bombardments.

\subsection{Desorption measurements of implanted Si(111) surfaces}

The desorption curves of implanted argon are shown in fig. 2, the steps appear in the order: $\sim 1030 \mathrm{~K}, \sim 840 \mathrm{~K}$ (main step - which is also the case
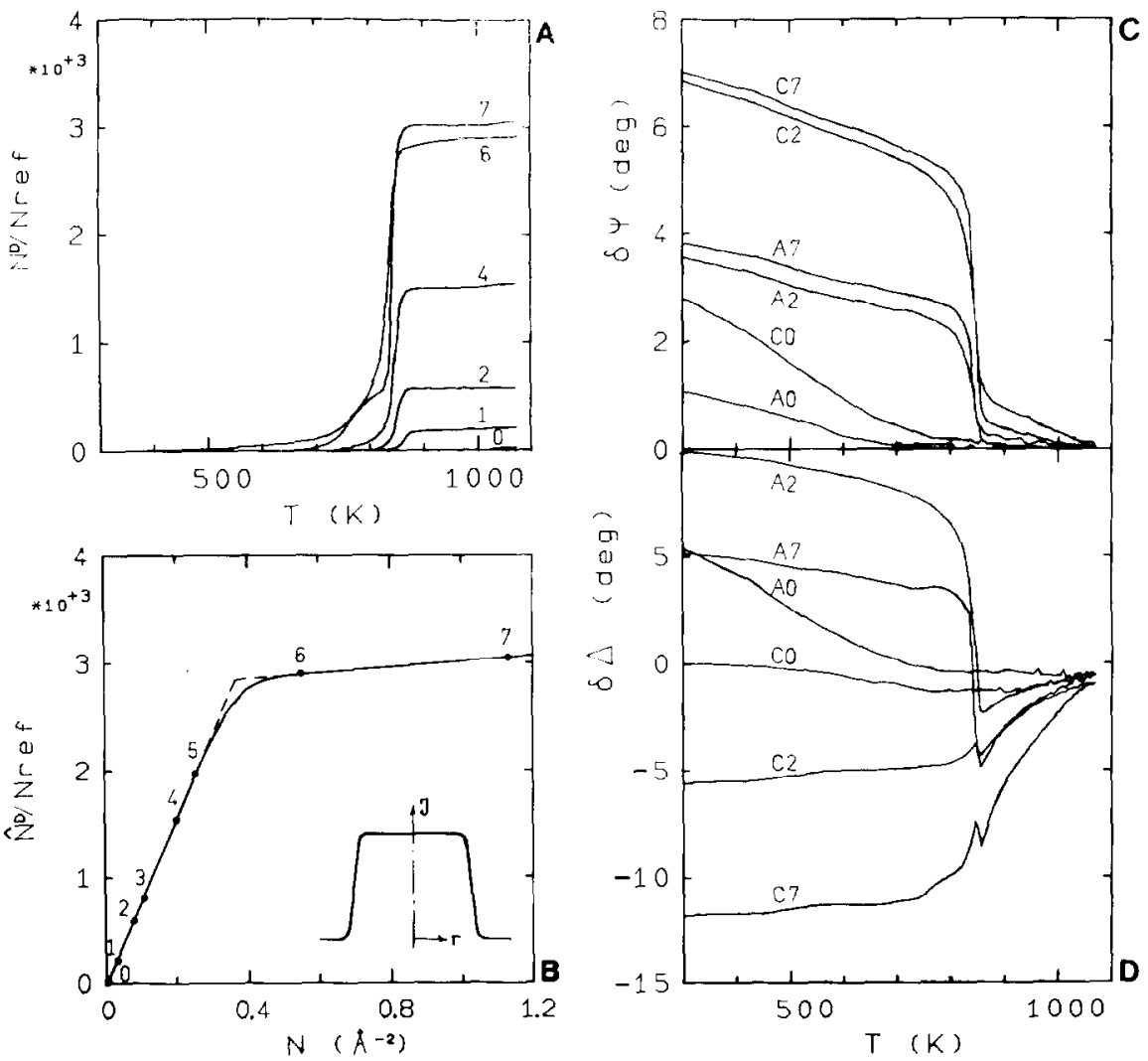

Fig. 2. Argon desorption experiments. (A) The desorbed dose as function of temperature. (B) The maximum desorbed dose at $1080 \mathrm{~K}$ as function of the bombardment dose. (C, D) $\delta \Delta$ and $\delta \psi$ as function of the desorption temperature at two wavelengths (A, C). The numbers $0-7$ correspond to the bombardment doses given at the top of fig. 1A. The insert in (B) gives the argon beam profile. 
for krypton!) and $\sim 780 \mathrm{~K}$, with increasing ion dose. The step at $\sim 1030 \mathrm{~K}$ appears after a very small dose, stays almost constant upon increasing dose, and is interpreted as being due to channelling. In fig. 2B the final desorbed doses $\hat{N}_{\mathrm{D}}$ are plotted as a function of the dose $N$; at $\sim 0.38 \AA^{-2}$ the sample is saturated with argon. The increase of $\hat{N}_{\mathrm{D}}$ after point 6 is partially due to the boundary of the beam profile. In figs. $2 \mathrm{C}$ and $2 \mathrm{D} \delta \Delta$ and $\delta \psi$ are given as a function of the sample temperature. Curves $\mathrm{A} 0$ and $\mathrm{C} 0$ exhibit monotonic damage recovery. At higher doses the large changes at $\sim 840 \mathrm{~K}$ are due to damage recovery and argon desorption, only above $840 \mathrm{~K}$ is the change in $\delta \Delta$ always positive. In fig. 3 the bombardment (flux 2 ) and desorption experiments are shown. For low doses the desorption curve retraces the bombardment curve $(2 \rightarrow 1)$. In general the slopes of the desorption curves are quite similar to those of the bombardment curves. From the curves in figs. 2C, 2D and 3, four different effects can be distinguished: section $3 \rightarrow 4$ damage recovery at room temperature; section $4 \rightarrow 5$, damage recovery due to increasing temperature; section $5 \rightarrow 6$, desorption of argon and a large damage recovery at $\sim 840 \mathrm{~K}$; section $6 \rightarrow 7$, complete recovery of the crystal. At $1080 \mathrm{~K}$ the crystal regains the original crystalline state after $\sim 30$ min annealing.

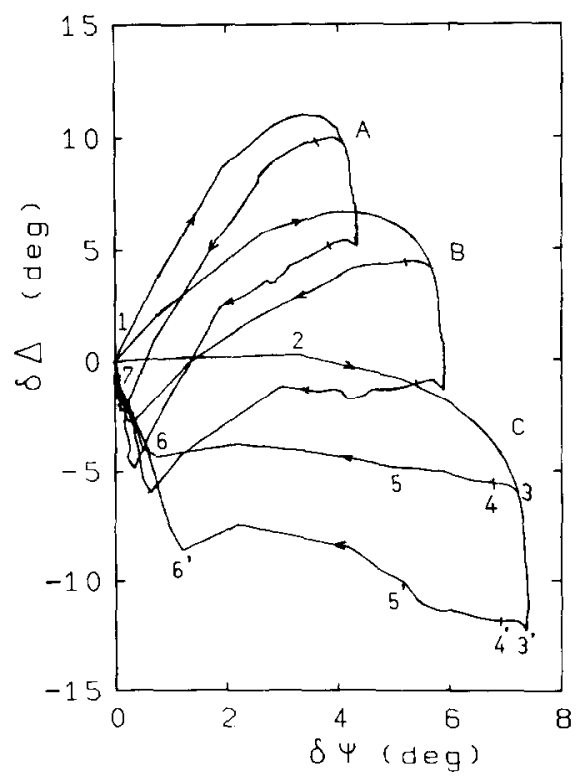

Fig. 3. $\delta \Delta-\delta \psi$ plot of argon bombardment (flux 2) and desorption experiments at three wavelengths A, B, C. Symbols 1-7: divisions of characteristic regions, explained in the text. 


\subsection{Discussion}

The complex dielectric constant, $\tilde{\boldsymbol{\epsilon}}$, of a mixture of components in a solid can be calculated with the EMA equation [6]. We assume a simple model of a homogeneous amorphous layer of variable thickness $(0-150 \AA)$ containing a particle fraction, $\theta_{1}$, of implanted argon $(0,0.05$ and 0.10$)$ above a crystalline substrate. The dielectric constant of argon $\left(\tilde{\epsilon}_{\mathrm{I}}=2.55-0 \mathrm{i}\right)$ was calculated using the Clausius-Mossotti equation [6]. The problem now arises which of the published $\tilde{\epsilon}_{\mathrm{a}}(\lambda)$ to use. Davis and Mott [11] propose that for amorphous silicon $(\alpha n E)^{1 / 3}=c\left(E-E_{0}\right)$, confirmed by other authors [1,12], where $c$ and $E_{0}$ are constants, $n$ is the index of refraction, $\alpha$ is the absorption coefficient and $E$ is the photon energy. From our measured $\delta \Delta$ and $\delta \psi$ values in the $E$ region $1.6-3.1 \mathrm{eV}$, after a dose $N=0.083 \AA^{-2}$, we calculate the amorphous layer thickness, $d$, under the condition that $(\alpha n E)^{1 / 3}$ is a linear function of $E$; from this calculation it follows that $E_{0}=1.05 \mathrm{eV}, c=5.45 \mathrm{eV}^{-2 / 3} \mu \mathrm{ml}^{-1 / 3}, d=100$ $\pm 5 \AA$, obtaining at the same time $\tilde{\epsilon}_{\mathrm{a}}(\lambda)$ (see fig. 4), where $\theta_{\mathrm{I}}$ is taken as 0.02 .

The results of the calculation for $\delta \Delta$ and $\delta \psi$ are shown in fig. 1C. For all wavelengths the measured values of $\delta \Delta$ and $\delta \psi$ for argon, at flux 2, can be explained by a layer of $\sim 85 \AA$ which amorphosises until point $\mathrm{Q}$ is reached,

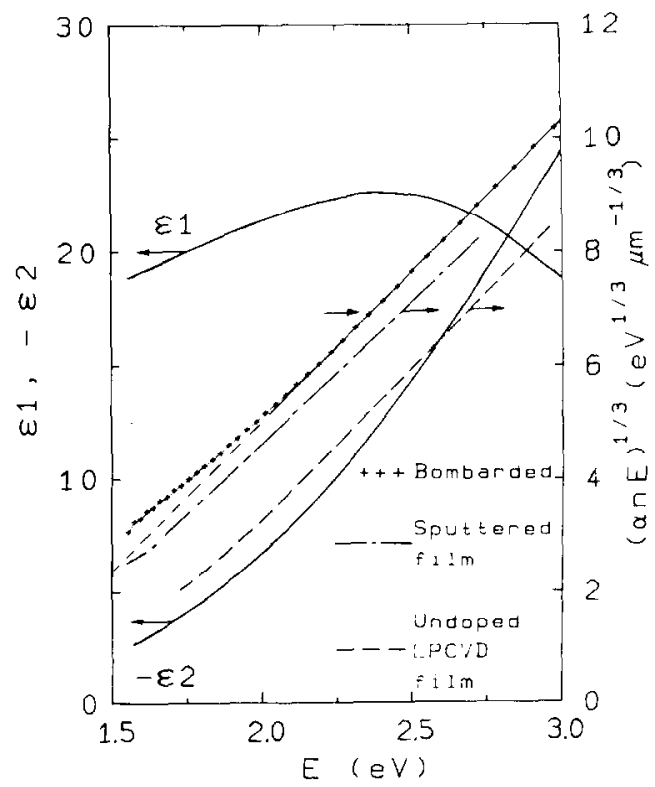

Fig. 4. The dielectric constant $\tilde{\epsilon}_{\mathrm{a}}(\lambda)$. A comparison with regard to the expression of Davis and Mott is made for an Ar-bombarded sample $(+++)$, a sputtered film (-·-) sample 1 from ref. [12], and an undoped LPCVD film (- - ) from ref. [1]. 
followed by a growth of the layer up to $\sim 140 \AA$ with $\theta_{\mathrm{I}} \simeq 0.10$. For the similar situation of krypton ion bombardment the values are $70 \AA$ and $\sim 105 \AA$ respectively.

At the dose of point $Q\left(0.03 \AA^{-2}\right)$ we compare the calculated amorphous layer thickness with the projected range, $\chi_{p}$, and range straggling, $\sigma_{p}$ [7], of $\operatorname{argon}\left(\chi_{p}=34.3 \AA, \sigma_{p}=19.9 \AA\right)$ and krypton $\left(\chi_{p}=38.3 \AA, \sigma_{p}=13.4 \AA\right)$ for 2 $\mathrm{keV}$ bombardment. One can expect an amorphous layer of $\chi_{\mathrm{p}}+3 \sigma_{\mathrm{p}}$, however, by using an layer of $\chi_{\mathrm{p}}+2.5 \sigma_{\mathrm{p}}$ the predicted thickness is $84 \AA$ for argon and $72 \AA$ for krypton, which is in agreement with the observed values.

Furthermore, from the ion collection model of Schulz and Wittmaack [8], using a sputter coefficient $s=1[9,10]$, one can calculate a maximum implanted argon dose of $1.7 \AA^{-2}$, while the measured value from the desorption experiments (fig. 2B) is $0.38 \AA^{-2}$. This means that the total amount of argon is limited by the solubility of argon in the silicon lattice, or $s \simeq 4$ finally.

By distributing the dose of $0.38 \AA^{-2}$ in a homogenous layer of $140 \AA$ one can calculate an argon concentration of $\theta_{\mathrm{I}}=0.05$ instead of $\theta_{1}=0.10$ in the final state. However for an accurate calculation one requires the actual distributions $\theta(z)$ and we have also omitted the surface roughness arising during bombardment. For the lower particle flux 1 it seems likely that the amorphous layer is not completely built up, as compared to flux 2 .

\section{References}

[1] D.E. Aspnes, A.A. Studna and E. Kinsbron, Phys. Rev. B29 (1984) 768.

[2] J.R. Adams and N.M. Bashara, Surface Sci. 49 (1975) 441.

[3] J.W.D. Martens, W.F. van den Bogert and A. van Silfhout, Surface Sci. 105 (1981) 275.

[4] K.O. van der Werf, R.J.S. Sieverdink, W. Vonk, G. Meijer and L.J. Hanekamp, to be published.

[5] A.H.M. Holtslag and A. van Silfhout, to be published.

[6] D.E. Aspnes, Am. J. Phys. 50 (8) (1982) 704.

[7] J.F. Gibbons, W.S. Johnson and S.W. Mylroie, Projected Range Statistics, Semiconductors and Related Material (Halsted, 1975).

[8] F. Schulz and K. Wittmaack, Radiation Effects 29 (1975) 31.

[9] K. Wittmaack and P. Blank, Appl. Phys. Letters 31 (1977) 21.

[10] R. Behrisch, Topics in Applied Physics 47 (Springer, Berlin, 1981) p. 169.

[11] E.A. Davis and N.F. Mott, Phil. Mag. 22 (1970) 903.

[12] R.H. Klazes, M.H.L.M. van den Broek, J. Bezemer and S. Radelaar, Phil. Mag. B45 (1982) 377. 